OPEN ACCESS

Edited by:

Amedeo D'Angiulli,

Carleton University, Canada

Reviewed by:

Ryan Donald Burns,

The University of Utah, United States

Cesar Cavinato Cal Abad,

NAR-Nucleus of High Performance

in Sport, Canada

*Correspondence:

Florian A. Engel

florian.enge/@issw.uni-heidelberg.de

Specialty section:

This article was submitted to

Children and Health,

a section of the journal

Frontiers in Public Health

Received: 13 May 2019 Accepted: 26 September 2019 Published: 14 November 2019

Citation:

Engel FA, Wagner MO, Schelhorn F Deubert F, Leutzsch S, Stolz A and Sperlich B (2019) Classroom-Based Micro-Sessions of Functional

High-Intensity Circuit Training

Enhances Functional Strength but Not Cardiorespiratory Fitness in School

Children-A Feasibility Study.

Front. Public Health 7:291.

doi: 10.3389/fpubh.2019.00291

\section{Classroom-Based Micro-Sessions of Functional High-Intensity Circuit Training Enhances Functional Strength but Not Cardiorespiratory Fitness in School Children-A Feasibility Study}

\author{
Florian A. Engel ${ }^{1 *}$, Matthias Oliver Wagner ${ }^{2}$, Franziska Schelhorn ${ }^{3}$, Felix Deubert ${ }^{3}$, \\ Sascha Leutzsch ${ }^{3}$, Alexander Stolz ${ }^{3}$ and Billy Sperlich ${ }^{3}$ \\ ${ }^{1}$ Department Movement and Training Science, Institute of Sport and Sport Science, Heidelberg University, Heidelberg, \\ Germany, ${ }^{2}$ Department of Sport Science, Bundeswehr University Munich, Neubiberg, Germany, ${ }^{3}$ Integrative and \\ Experimental Training Science, Institute for Sport Sciences, University of Würzburg, Würzburg, Germany
}

The present study assessed the short-term effect of 6 min classroom-based micro-sessions of multi-joint functional high-intensity circuit training (Functional$\left.\right|_{H I T}$ ) performed by students during regular classes on parameters related to functional strength and cardiorespiratory fitness. In this randomized controlled 4-week study, 17 students (11 male; 6 female; age: $11.6 \pm 0.2$ years) performed 6 min of Functional $\left.\right|_{H I T}$ (targeting $>17$ on the Borg scale) 4 days per week during regular school classes and 18 students (11 male; 7 female; age: $11.7 \pm 0.3$ years) served as control group (CG) without any additional in-class physical activity. The Functional ${ }_{H I T}$ group completed $86 \%$ of all planned sessions (mean duration: $6.0 \pm 1.5 \mathrm{~min}$ ) with a mean RPE of $17.3 \pm 2.1$. Body height, mass and BMI did not differ between the groups at baseline or between pre- and post-testing $\left(p>0.05\right.$; eta $\left.{ }^{2} \leq 0.218\right)$. The performances in lateral jumping $(p<0.000$; part eta $\left.{ }^{2}=0.382 ; \Delta \% 4.6 \pm 8.6\right)$, sit-ups $\left(p<0.000 ;\right.$ part eta ${ }^{2}=0.485 ; \Delta \% 3.1 \pm$ 8.6) and 20-m sprints ( $p<0.000$; part eta ${ }^{2}=0.691 ; \Delta \% 15.8 \pm 5.4$ ) improved in both groups with greater increase following Functional $\left.\right|_{H I T}$. No baseline differences and no interaction effects occurred in performance of 6 min run, flexibility, push-ups, balance,

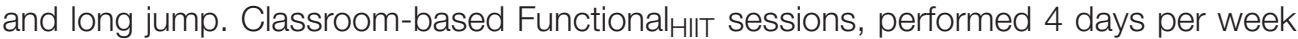
during 4 weeks did not improve variables related to aerobic endurance performance but enhanced certain parameters of functional strength in schoolchildren. As time is limited in the educational system of schools, Functional $\left.\right|_{H I T}$ during regular school classes could offer a new perspective for increasing functional strength in schoolchildren.

Keywords: aerobic fitness, exercise intervention, functional training, interval training, physical activity promotion 


\section{INTRODUCTION}

Youth levels of physical inactivity is increasing in Europe (1) and worldwide (2) with $70-90 \%$ of European adolescents failing recommended levels of physical activity (PA) (2) and with high sedentary behavior (1). A low level of PA may result in poor cardiorespiratory fitness (CRF) while low PA and CRF are both considered as risk factors for developing cardiovascular diseases (CVD) (3-5) and obesity (3-6). While most recommendations for regular PA of children and adolescents refer to moderateto-vigorous activity of $60 \mathrm{~min}$ daily (7), repeated bouts of highintensity exercise [i.e., high-intensity interval training (HIIT)] demonstrated time-efficient (8-10) and positive effects for improving CRF in (non-)obese children and adolescents with most interventions ranging from 7 to 10 weeks with $2-3$ sessions per week (9-13).

Since children spend $40 \%$ of their waking hours in the school (14) it seems the school setting is ideal to implement PA promotion. In this context HIIT was recently applied within physical education $(\mathrm{PE})$ classes of children $(15,16)$ and adolescent students $(17,18)$ to enhance CRF [determined by a shuttle run $(17,18)$ and 6 min walking test $(16)]$. Additionally, HIIT not only improved CRF but also functional strength when applied for relative short periods of 7-10 weeks with 13 sessions/week $(19,20)$. PE pursues additional objectives other than exclusively improving CRF, functional strength or motor performance (i.e., sport specific skill acquisition, motor learning, and development of personal-, social- and methodological competences). Thus, PE as school subject per se and due to time constraints seems restricted to improve CRF, functional strength and motor performance sustainably. Because of its proven time-efficiency and effectiveness in improving the key variables CRF and short functional strength bouts of HIIT could be applied during or in-between regular (non PE) classes within the academic classroom sparing enough time for other academic aims.

Usually HIIT protocols are running- (20-23) or cyclingbased $(22,24,25)$, requiring either space or equipment. As a special form of HIIT circuit-like, multiple-joint, high-intensity exercises (Functional ${ }_{\mathrm{HIIT}}$ ), performed daily during 4 weeks (26), respectively three sessions per week during 9 weeks (27), have been applied successfully to improve CRF and functional strength, body composition as well as certain aspects of quality of life in adults. Functional HIIT $_{\text {does not require a }}$ lot of space or equipment and is therefore applicable in any academic classroom. A recent large scale study demonstrated that, independent of the time and patterns of sedentary behavior, moderate-to-vigorous physical activity is associated with health-related physical fitness in 13-year old children (28). Therefore, we assume that micro-sessions of Functional HIT $_{\text {T }}$ may contribute to improve health-related physical fitness and ultimately reduce the risk for cardiovascular diseases in children

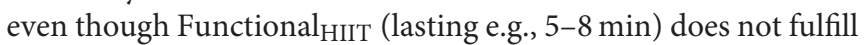
the current recommendations for PA. Although time-efficient 6 min Functional $_{\text {HIIT }}$ proved to increase muscular strength and perceived quality of life in untrained adults (26) it is astonishing that this type of exercise has not been applied in an academic classroom during school hours to evaluate whether Functional HIT $_{\text {improves CRF and functional strength in school }}$ children. Therefore, the aim of this study was to evaluate the effects of a $6 \mathrm{~min}$ circuit-like, multiple-joint high-intensity interval training performed within regular school classes in the academic classroom of 11-12 year old school children vs. a group performing no additional physical activity in the classroom.

The potential benefits of implementing Functional $_{\text {HIIT }}$ interventions in the academic classroom include (i) bypassing "lack of time" as one reason for not exercising (29) and (ii) the high degree of adherence to the PA interventions at school.

We hypothesized that the Functional HIIT $_{\text {group would }}$ significantly improve cardiorespiratory fitness and functional strength from baseline $\left(\mathrm{T}_{0}\right)$ to after the four-week intervention $\left(\mathrm{T}_{1}\right)$ in comparison to the control group.

\section{MATERIALS AND METHODS}

\section{Participants}

In this single-center, two-arm randomized, controlled study, $n=35$ secondary school children (24 male; 11 female; age: 11.7 \pm 0.3 years) of the south of Germany participated (Table $\mathbf{1}$ ).

During the 4 week intervention, $n=17$ (11 male; 6 female; age: $11.6 \pm 0.2$ years) students performed 4 days per week one supervised 6 min micro-session of Functional HIIT (Table 2) during their regular school class and 18 students (11 male; 7 female; age: $11.7 \pm 0.3$ years) served as control group without additional in-class exercise. All participants were free to withdraw from the study at any time without further consequences. The inclusion criteria were: (i) an age of 10-12 years; (ii) no frequent participation in endurance or strength exercise programs for at least 6 months prior to the study; (iii) no daily intake of medication; and (iv) for inclusion in the analysis, completion of at least $80 \%$ of all possible training sessions.

All procedures were conducted in accordance with the Declaration of Helsinki (30). The experimental protocol was pre-approved by the ethical review board of the Sport Science Institute of the University of Würzburg. All students and their legal guardians as well as their teachers were informed in detail about the design of the study, including the potential risks and benefits, before providing their written informed consent to participate.

\section{Overall Study Design}

At baseline $\left(T_{0}\right)$ and after the four-week intervention $\left(T_{1}\right)$, all students underwent assessment of body height and mass. Functional strength and CRF were assessed using the "German Motor Ability Test" (31). The post-intervention assessment $\left(\mathrm{T}_{1}\right)$ took place $72 \mathrm{~h}$ after the final scheduled Functional ${ }_{\text {HIT }}$ session (Figure 1). All assessments during $\mathrm{T}_{0}$ and $\mathrm{T}_{1}$ were performed in the gym and track and field court of the school, supervised by the principal investigators, teacher and trained undergraduate students. The German Motor Ability Test shows a very good independence from the examiner, with a mean correlation coefficient for all eight tests of $r=0.95$ (range: $0.86-0.99$ ). TestRetest reliability shows a mean correlation coefficient for all eight tests of $r=0.82$ (31). 
TABLE 1 | Anthropometric parameters (means \pm SD) for the Functional ${ }_{\text {HIIT }}(n=17)$ and control group $(n=18)$ before $\left(T_{0}\right)$ and after $\left(T_{1}\right)$ the 4-week intervention.

\begin{tabular}{|c|c|c|c|c|c|c|c|c|c|}
\hline Parameter & Group & $T_{0}$ & $\mathrm{~T}_{1}$ & $p$ & $\eta^{2}$ & $\mathbf{F}$ & $p$ & $\eta^{2}$ & $\mathbf{F}$ \\
\hline & & & & & (T) & & & $(T \times G)$ & \\
\hline \multirow[t]{2}{*}{ Body height [m] } & Functional $\left.\right|_{H I T}$ & $1.57 \pm 0.07$ & $1.58 \pm 0.06$ & 0.424 & 0.021 & 0.801 & 0.104 & 0.011 & 0.415 \\
\hline & Control & $1.58 \pm 0.07$ & $1.58 \pm 0.07$ & & & & & & \\
\hline \multirow[t]{2}{*}{ Body mass [kg] } & Functional $_{\text {HIIT }}$ & $40.7 \pm 7.0$ & $41.3 \pm 6.7$ & 0.725 & 0.015 & 0.327 & 0.676 & 0.004 & 0.182 \\
\hline & Control & $46.0 \pm 10.0$ & $46.2 \pm 9.9$ & & & & & & \\
\hline \multirow[t]{2}{*}{ Age [years] } & Functional $_{\text {HIIT }}$ & $11.6 \pm 0.2$ & $11.6 \pm 0.2$ & - & - & - & - & - & - \\
\hline & Control & $11.7 \pm 0.3$ & $11.7 \pm 0.3$ & & & & & & \\
\hline
\end{tabular}

p, probability; $\eta^{2}$, effect size partial eta-square; T, global effect of time; $T \times G$, global effect of Time $\times$ Group; $F$, degrees of freedom.

The school was chosen since the school offered a sufficient number of students and one researcher had contact to the school before the start of the study.

\section{Functional $_{\text {HIT }}$}

In week 1 participants performed three training sessions, during week 2-4 participants performed four Functional ${ }_{\text {HIT }}$ sessions per week (Table 2). During the 4-week intervention period participants performed different $6 \mathrm{~min}$ micro-sessions of Functional HIIT (Table 2) in the academic classroom during school hours. Functional ${ }_{\text {HIT }}$ involved $6 \mathrm{~min}$ circuit-like, multiple-joint exercises with high-intensity interval training protocols and all exercises were performed with the children's own bodyweight and rather high velocities and repetitions (Table 2). During each Functional HIIT $_{\text {session the teacher }}$ demonstrated Functional ${ }_{\text {HIT }}$ movements (for details, see Table 2 and www.sportsandscience.de, 2017a; b; c; d). The children were instructed to follow these movements with "allout" effort targeting $>17$ on the $6-20$ Borg scale (32). Children aged 11-14 demonstrated being able to regulate their exercise intensity independently to correspond to intensities of 17 (very hard) using the 6-20 Borg scale (33). The teacher gave feedback to students considering the correct execution of Functional ${ }_{\text {HIT }}$ movements and provided standardized verbal encouragement.

\section{Anthropometric Data and Body Composition}

Height was measured with a portable stadiometer (seca 213, seca, Hamburg, Germany). Body mass was assessed with an electronic scale (seca 799, seca, Hamburg, Germany). Height and body mass were measured while participants wore sports clothes and no shoes. The body-mass-index was calculated as body mass/(body height) ${ }^{-2}$.

\section{German Motor Ability Test}

The German Motor Ability Test was performed as described in detail elsewhere (31) and has been applied frequently to assess $\mathrm{CRF}$ and functional strength (34-36). As mentioned above, the German Motor Ability Test shows very good test quality criteria for all test items (31). This type of testing is frequently applied in German PE so that all children of the present study were accustomed to all procedures. Briefly, the German Motor Ability Test comprises of eight different subtests: (i) 20- $m$ sprint: All participants sprinted a 20-m distance as fast as possible. Time was measured with single beam timing lights (Brower Timing Systems, Draper, USA) and used for statistical analysis.

(ii) Standing long jump: All children jumped once from standing position with both legs as far as possible. The covered distance employed for statistical analysis.

(iii) Forward bend: All children stood on a bench and bended their trunk forward toward their feet with straight elbows and knees. The deepest position of the fingertips was obtained for statistical analysis.

(iv) Lateral jumping: Participants completed within $15 \mathrm{~s}$ as many lateral jumps from one side of a square $(50 \times$ $100 \mathrm{~cm}$ ) to another. The number of correctly performed jumps was employed for statistical analysis.

(v) Balancing backwards: All children balanced backwards on bars of three different widths (consecutively: 6.0, 4.5, and $3.0 \mathrm{~cm}$ ). The number of steps before touching the ground (maximum $n=8$ ) per bar were obtained for statistical analysis.

(vi) Sit-Ups: All performed as many sit-ups as possible within 40-s. The number of correct repetitions within 40-s were counted for statistical analysis.

(vii) Push-ups: Participants performed as many push-ups as possible within 40 -s. The number of correctly performed repetitions within 40-s were counted for statistical analysis.

(viii) 6 min run: All participants ran or walked constantly around a volleyball court $(9 \times 18 \mathrm{~m})$ for $6 \mathrm{~min}$. The covered distance of each participant was obtained for statistical analysis.

\section{Ratings of Perceived Exertion}

Participants were familiarized with the Borg 6-20 RPE scale before the start of the intervention (32). Immediately after each Functional $_{\text {HIT }}$ session, all students rated their perceived exertion during exercise on the Borg 6-20 scale (32). Children aged $\sim 11$ years were found capable of expressing exercise intensities on the RPE scale as precise as adults (37). For analysis, we averaged the data from all participants over all Functional ${ }_{\text {HIT }}$ sessions. Before the start of the study we assess the heart rate response of ten children during Functional ${ }_{\text {HIT }}$ to ensure the heart rate would increase to $>85 \%$ of heart rate max. Since we could 
TABLE 2 | Details of the 4-week Functional $\left.\right|_{\text {HIIT }}$ training intervention.

\begin{tabular}{|c|c|c|}
\hline Week & Session & Functional $_{H I I T}$ \\
\hline \multirow[t]{3}{*}{1} & 1 & $\begin{array}{l}-2 \text { series of } \\
\quad-30 \text {-s squats }+15 \text {-s recovery } \\
\quad-30 \text {-s lunges }+15 \text {-s recovery } \\
-3 \text { series of } \\
\quad-50 \text {-s skippings }+10 \text {-s recovery }\end{array}$ \\
\hline & 2 & $\begin{array}{l}-2 \text { series of } \\
\quad-20 \text {-s push-ups }+10 \text {-s recovery } \\
\quad-20 \text {-s crunches }+10-\text {-s recovery } \\
\quad-20 \text {-s dips }+10 \text {-s recovery } \\
-3 \text { series of } \\
\quad-50 \text {-s skippings }+10 \text {-s recovery }\end{array}$ \\
\hline & 3 & $\begin{array}{l}\text { - } 45 \text {-s jumping jacks }+15 \text {-s recovery } \\
-45 \text {-s plank }+15 \text {-s recovery } \\
-2 \text { series of } \\
\quad-20 \text {-s burpees }+10 \text {-s recovery } \\
-3 \text { series of } \\
\quad-50 \text {-s skippings }+10 \text {-s recovery }\end{array}$ \\
\hline \multirow[t]{4}{*}{2} & 1 & $\begin{array}{l}-2 \text { series of } \\
\quad-30 \text {-s squats }+15 \text {-s recovery } \\
\quad-30 \text {-s lunges }+15 \text {-s recovery } \\
-3 \text { series of } \\
\quad-50 \text {-s skippings }+10 \text {-s recovery }\end{array}$ \\
\hline & 2 & $\begin{array}{l}-2 \text { series of } \\
\quad-20 \text {-s push-ups }+10-\text {-s recovery } \\
\quad-20 \text {-s crunches }+10 \text {-s recovery } \\
\quad-20 \text {-s dips }+10 \text {-s recovery } \\
-3 \text { series of } \\
\quad-50 \text {-s skippings }+10 \text {-s recovery }\end{array}$ \\
\hline & 3 & $\begin{array}{l}-45 \text {-s jumping jacks }+15 \text {-s recovery } \\
-45 \text {-s plank }+15 \text {-s recovery } \\
-2 \text { series of } \\
\quad-20 \text {-s burpees }+10 \text {-s recovery } \\
-3 \text { series of } \\
\quad-50 \text {-s skippings }+10 \text {-s recovery }\end{array}$ \\
\hline & 4 & $\begin{array}{l}-2 \text { series of } \\
\quad-30 \text {-s squats }+15 \text {-s recovery } \\
\quad-30 \text {-s lunges }+15 \text {-s recovery } \\
-3 \text { series of } \\
\quad-50 \text {-s skippings }+10 \text {-s recovery }\end{array}$ \\
\hline \multirow[t]{4}{*}{3} & 1 & $\begin{array}{l}-2 \text { series of } \\
\quad-20 \text {-s push-ups }+10 \text {-s recovery } \\
\quad-20 \text {-s crunches }+10 \text {-s recovery } \\
\quad-20 \text {-s dips }+10 \text {-s recovery } \\
-3 \text { series of } \\
\quad-50 \text {-s skippings }+10 \text {-s recovery }\end{array}$ \\
\hline & 2 & $\begin{array}{l}\text { - } 45 \text {-s jumping jacks }+15 \text {-s recovery } \\
-45 \text {-s plank }+15 \text {-s recovery } \\
-2 \text { series of } \\
\quad-20 \text {-s burpees }+10 \text {-s recovery } \\
-3 \text { series of } \\
\quad-50 \text {-s skippings }+10 \text {-s recovery }\end{array}$ \\
\hline & 3 & $\begin{array}{l}-2 \text { series of } \\
\quad-30 \text {-s squats }+15 \text {-s recovery } \\
\quad-30 \text {-s lunges }+15 \text {-s recovery } \\
-3 \text { series of } \\
\quad-50 \text {-s skippings }+10 \text {-s recovery }\end{array}$ \\
\hline & 4 & $\begin{array}{l}-2 \text { series of } \\
\quad-20 \text {-s push-ups }+10 \text {-s recovery } \\
\quad-20 \text {-s crunches }+10 \text {-s recovery } \\
-20 \text {-s dips }+10 \text {-s recovery } \\
-3 \text { series of } \\
\quad-50 \text {-s skippings }+10 \text {-s recovery }\end{array}$ \\
\hline
\end{tabular}

(Continued)
TABLE 2 | Continued

\begin{tabular}{|c|c|c|}
\hline Week & Session & Functional $_{H I I T}$ \\
\hline \multirow[t]{4}{*}{4} & 1 & $\begin{array}{l}-45 \text {-s jumping jacks }+15 \text {-s recovery } \\
-45 \text {-s plank }+15 \text {-s recovery } \\
-2 \text { series of } \\
\quad-20 \text {-s burpees }+10 \text {-s recovery } \\
-3 \text { series of } \\
\quad-50 \text {-s skippings }+10 \text {-s recovery }\end{array}$ \\
\hline & 2 & $\begin{array}{l}-2 \text { series of } \\
\quad-30 \text {-s squats }+15 \text {-s recovery } \\
\quad-30 \text {-s lunges }+15 \text {-s recovery } \\
-3 \text { series of } \\
\quad-50 \text {-s skippings }+10 \text {-s recovery }\end{array}$ \\
\hline & 3 & $\begin{array}{l}-2 \text { series of } \\
\quad-20 \text {-s push-ups }+10 \text {-s recovery } \\
\quad-20 \text {-s crunches }+10-\text {-s recovery } \\
\quad-20 \text {-s dips }+10-\text {-s recovery } \\
-3 \text { series of } \\
\quad-50 \text {-s skippings }+10 \text {-s recovery }\end{array}$ \\
\hline & 4 & $\begin{array}{l}-2 \text { series of } \\
\quad-30 \text {-s jumping jacks }+15 \text {-s recovery } \\
\quad-30 \text {-s squats }+15 \text {-s recovery } \\
\quad-3 \text { series of } \\
\quad-50 \text {-s skippings }+10 \text {-s recovery }\end{array}$ \\
\hline
\end{tabular}

not apply heart rate monitoring during regular school classes (due to time constraints) we employed RPE as surrogate for the training intensity.

\section{Statistical Analyses}

All data of $\mathrm{T}_{0}$ and $\mathrm{T}_{1}$ were confirmed to be normally distributed by the Kolmogorov-Smirnov test and homogeneity of variance (Levene Test) were tested prior to further statistical analysis, so that no transformation was required. A two way repeatedmeasures ANOVA [time-point $\left(\mathrm{T}_{0}, \mathrm{~T}_{1}\right) \times$ group (Functional ${ }_{\mathrm{HIT}}$, CG)] was performed for each outcome variable, with an alpha of $p<0.05$ being considered statistically significant and indicated by asterisk. The values obtained were evaluated by calculating the effect size partial eta-square $\left(\eta^{2}\right)(38)$ in order to evaluate practical relevance, with $\eta^{2} \geq 0.01$ indicated small, $\geq 0.06$ medium and $\geq 0.14$ large effects (39). Additionally, data of $\mathrm{T}_{0}$ was checked for significant differences between the two groups, applying student's $t$-test for unpaired samples. $G^{*}$ power software (40) was used to calculate the required sample size and based on the study of Sperlich et al. (26) it was determined that 20 participants per group would be required to provide sufficient power to detect statistically significant effects. The means and standard deviations $(S D)$ for all data sets were calculated and all statistical tests were carried out in SPSS 22.0 software (SPSS Inc., Chicago, IL, USA).

\section{RESULTS}

\section{Training Adherence, Training Duration, Perceived Exertion}

All students of the Functional ${ }_{\text {HIT }}$ group achieved the required minimum of $80 \%$ of scheduled training with $86 \%$ sessions completed during the 4-week intervention. No participant dropped out during the 4-weeks intervention. Two participants 


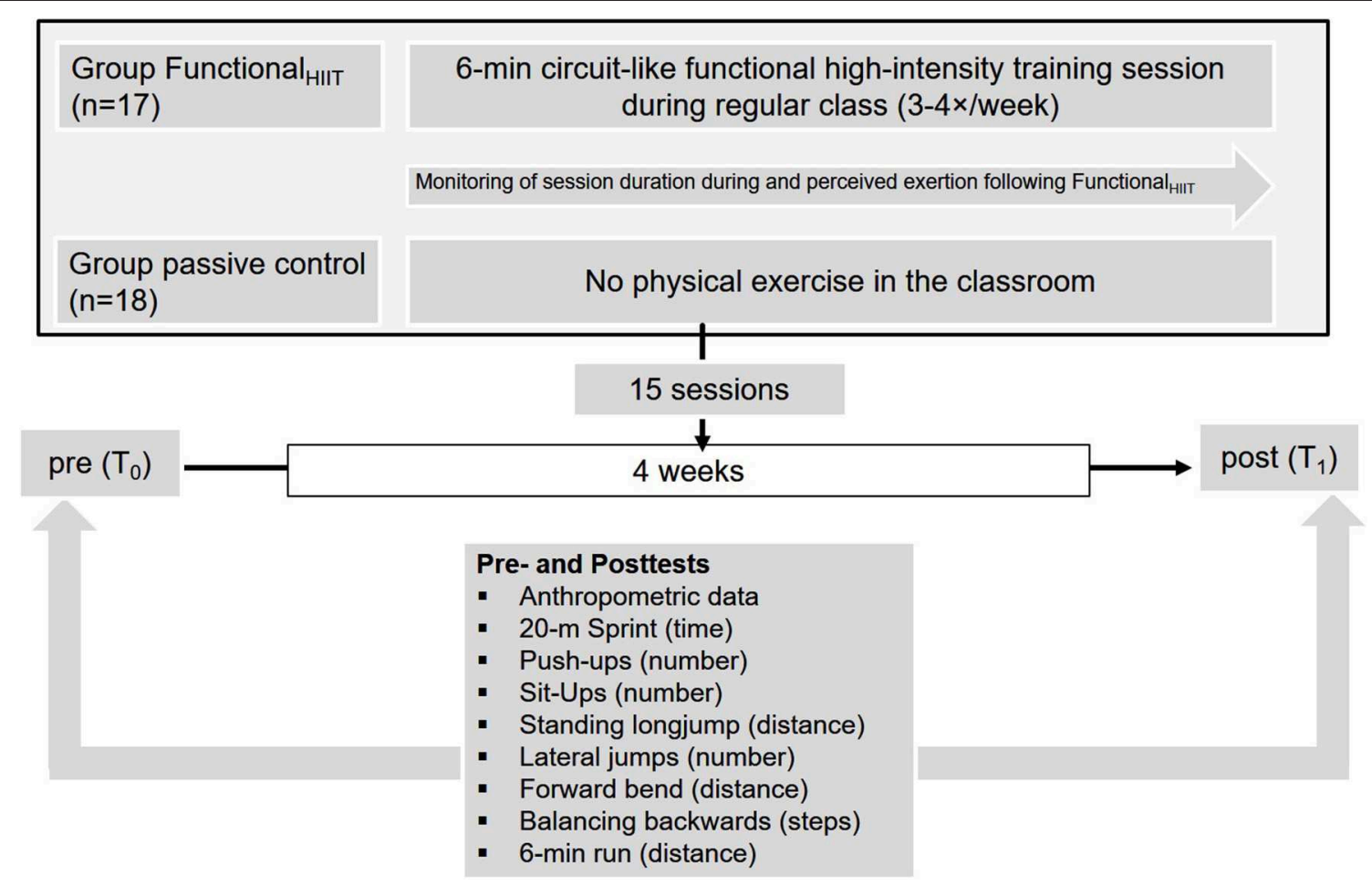

FIGURE 1 | The study design, including testing prior to $\left(T_{0}\right)$ and following the intervention period $\left(T_{1}\right)$.

were excluded from analyses due to absence during the posttesting. The mean duration of Functional ${ }_{\text {HIIT }}$ sessions was $6.0 \pm$ $1.5 \mathrm{~min}$, the corresponding mean value for RPE was $17.3 \pm 2.1$.

\section{Pre-post Testing}

All parameters obtained at $\mathrm{T}_{0}$ and $\mathrm{T}_{1}$ and statistical analyses are presented in Tables 1, 3. All parameters measured at $\mathrm{T}_{0}$ did not differ between the two groups (lowest $p \geq 0.113$; lowest $\eta^{2} \geq$ 0.003). All anthropometric data did not differ between $T_{0}$ to $T_{1}$ and groups (lowest $p \geq 0.104$; lowest $\eta^{2} \geq 0.004$ ).

The performances in lateral jumping [effect of time $\times$ group: $F_{(1,33)}=20.40 ; p<0.000$; part eta ${ }^{2}=0.382$ ], sit-ups [effect of time $\times$ group: $F_{(1,33)}=31.13 ; p<0.000$; part eta $\left.{ }^{2}=0.485\right)$ and $20-\mathrm{m}$ sprint [effect of time $\times$ group: $F_{(1,33)}=73.69 ; p$ $<0.000$; part eta $\left.^{2}=0.691\right)$ increased in both groups with greater improvements in Functional ${ }_{\text {HIT }}$ vs. control group. All interactions revealed large effect sizes $\left(\mathrm{eta}^{2}=0.382-0.691\right)$. The total difference in performance at $\mathrm{T}_{1}$ between Functional $\mathrm{HIIT}_{\mathrm{T}}$ and control group (difference in performance $=$ performance

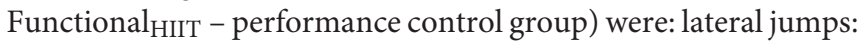
4.9 [n]; sit-ups: 3.0 [n]; 20-m sprint: 0.65 [s].

The performance in $6 \mathrm{~min}$ run [effect of time $\times$ group: $\mathrm{F}_{(1,33)}=0.258 ; p<0.615$; part eta $^{2}=0.008$ ], flexibility [effect of time $\times$ group: $F_{(1,33)}=3.974 ; p<0.055$; part eta $\left.{ }^{2}=0.107\right]$, push-ups [effect of time $\times$ group: $F_{(1,33)}=0.576 ; p<0.453$; part eta $^{2}=0.017$ ), balance [effect of time $\times$ group: $F_{(1,33)}=0.000 ; p$ $<0.992$; part eta ${ }^{2}=0.000$ ) as well as standing long jump [effect of time $\times$ group: $F_{(1,33)}=0.499 ; p<0.485$; part eta $\left.^{2}=0.015\right)$ revealed no significant changes over time and between groups (see Table 3). The absolute difference in performance between Functional ${ }_{\text {HIT }}$ and control group was: 6 min run: 80.0 [m]; flexibility: 0.8 [cm]; push-ups: $1.0[\mathrm{n}]$; balance: 0.2 [steps]; standing long jump: $5.0[\mathrm{~cm}]$.

\section{DISCUSSION}

The main findings of the present study concerning the responses of variables related to functional strength and CRF of untrained 11-year-old students to either 4 weeks of classroom-based Functional ${ }_{\text {HIT }}$ or passive control condition with no exercise were as follows:

(i) performance in lateral jumping, sit-ups and 20-m sprint were greater after 4 weeks Functional ${ }_{\text {HIIT }}$ compared to the control group;

(ii) in both groups no changes occurred after 4 weeks in 6 min run, flexibility, push-ups, balance as well as standing long jump;

(iii) all anthropometric parameters remained unchanged between groups and over time.

In contrast to CRF, the parameters related to functional strength (lateral jumping, sit-ups, 20-m sprint) exhibited significant interaction effects in favor of Functional ${ }_{\text {HIIT }}$ group, demonstrating the effectiveness of Functional ${ }_{\text {HIT }}$ for the improvement of functional strength. Since muscular fitness is inversely associated with cardiovascular disease risk factors and improvements in muscular fitness and sprinting speed seem to demonstrate a positive effect on skeletal health in children and 
TABLE 3 | Parameters of functional strength and endurance (means \pm SD) for the Functional $H_{H I T}$ and Controle group before $\left(T_{0}\right)$ and after $\left(T_{1}\right)$ the 4 week intervention.

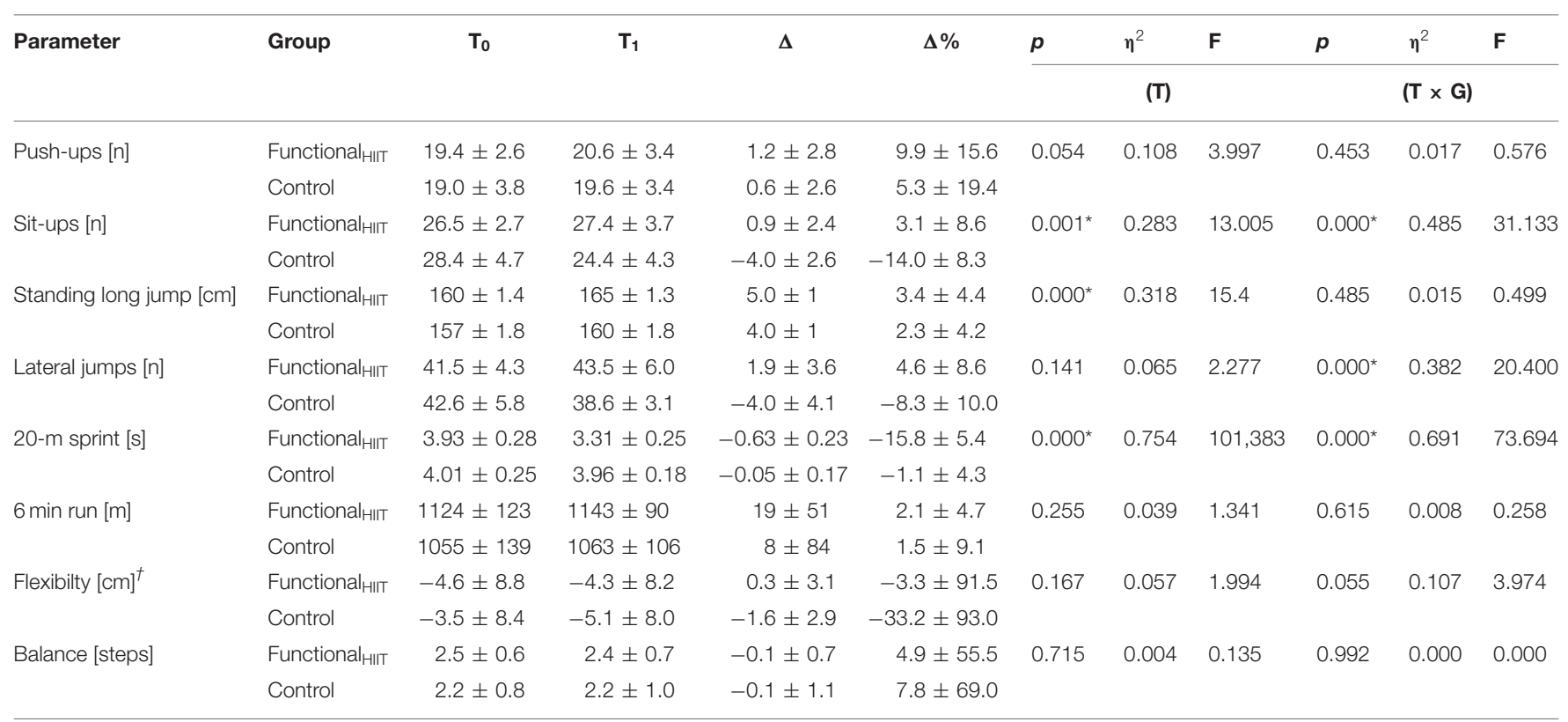

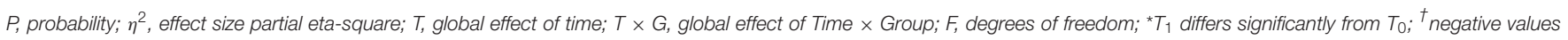
indicate a better flexibilty.

adolescents (3), the present Functional ${ }_{\text {HIIT }}$ intervention may reveal beneficial health effects. Overall, following Functional ${ }_{\text {HIIT }}$ the adaptations in functional strength (lateral jumping, sit-ups, 20-m sprint) were higher than those of CRF. These finding are in line with other studies performing similar HIIT sessions with children at school $(41,42)$ as well as with adults in other settings $(26,27)$. These findings underline that functional strength performance is responsive for adaptions following a short exercise intervention such as the present Functional HIIT $_{\text {. }}$ The pronounced increase in functional strength following Functional $\mathrm{HIIT}_{\mathrm{T}}$ may be due to adaptations in neuro-muscular structure and function, e.g., release of inhibitory mechanisms, as well as improvements in intra- and intermuscular coordination (synchronization, recruitment, and the rate coding of muscle fibers) with improved neural adaptations $(43,44)$. It seems that the short duration and the four training sessions per week of Functional HIIT $_{\text {are }}$ a potent stimulus to promote equivalent increases in neuro-muscular function when compared to other traditional strength training programs (45).

The present results are consistent with other findings demonstrating the positive impact of power and strength training on functional strength as well as on jumping and sprinting performance in children and adolescents in different settings including physical education $(44,46,47)$.

Surprisingly, the control group also demonstrated improvements from $\mathrm{T}_{0}$ to $\mathrm{T}_{1}$ for some test items (e.g., push-ups: 5.3\%; standing long jump: 2.3\%; flexibility: $33.2 \%$; balance: $7.8 \%)$. Since the control group performed no additional physical exercise during the classes, it is possible that improvements occured due to adaptations to regular physical education classes, extracurricular sports engagement or due to developmental effects. However, since all anthropometric parameters remained unchanged between groups and over time it is unlikely that developmental effects caused the improvements. Since the German Motor Ability Test demonstrates a high mean correlation coefficient for all eight test items of $r=0.82$ (31), we suggest that it is more likely that improvements of the control group arose from regular PE classes or extracurricular sports engagement. However, since the study did not control for PA level we cannot identify the cause for performance improvements with certainty.

From a practical perspective and in contrast to traditional apparatus-based strength training the present Functional ${ }_{\text {HIIT }}$ did not require any barbells, devices or machinery. All exercises were performed with the children's own bodyweight and rather high velocities and repetitions. The changes in functional strength after 4 weeks represent a good cost-benefit ratio since no expensive equipment was necessary-a prerequisite for school-based sports activities. Since the space requirement for Functional ${ }_{\text {HIT }}$ is very low (all exercises comprised mainly vertical movements in an area of about $1 \mathrm{~m}^{2}$ per student) the chosen Functional ${ }_{\text {HIIT }}$ in connection with the low space requirements favor this type of exercise as classroom based PA.

Despite the current HIIT-related research in children and adolescents $(9,10,12,13,48)$ only few classroom- or schoolbased HIIT micro-sessions have been examined with scientific rigor $(41,42)$. Therefore, it is difficult to determine which components of HIIT protocols [e.g., interval intensity and duration, rest intensity and duration, exercise modality, number of repetitions, number of series, between-series recovery duration and intensity (49)] maybe superior for improving dimensions of functional strength and CRF of young students. 
In contrast to other studies dealing with HIIT and young and adolescent students in a school-based environment $(16,17,20$, $21,41,48,50,51)$ the present study demonstrated no significant effects on CRF (6 min run performance) by Functional ${ }_{\mathrm{HIT}}$ and several reasons may explain this result. The short session durations of Functional ${ }_{\text {HIT }}(6.0 \pm 1.5 \mathrm{~min})$ combined with the short intervention period (4 weeks) as well as the exercise mode of Functional ${ }_{\text {HIT }}$ (i.e., the movements were more related to functional strength) may not have evoke a sufficient stimulus to improve CRF in the participating children. Other school-related HIIT studies improved peak oxygen uptake and employed a longer intervention periods ( $\geq 6$ weeks) and/or session durations $(10,15,19,34,40,41)$ ranging from approximately $14 \mathrm{~min}$ (17), $40 \mathrm{~min}(10,15,40)$ up to $60 \mathrm{~min}(20)$. In a recent review Braaksma et al. (11) recommended for improving CRF with PA interventions in children from 6 to 12 years a duration of at least 6 weeks and a frequency of 3-4 times per week. Most probably, our intervention did not improve CRF because each Functional ${ }_{\text {HIT }}$ sessions and overall intervention period were too short. While an extension of the present intervention period (4 weeks) seems feasible, we consider an extension of session duration (6.0 \pm $1.5 \mathrm{~min}$ ) of classroom-based interventions as unfavorable.

In the light of the present results, we cannot recommend $6 \mathrm{~min}$ Functional $_{\text {HIIT }}$ in order to improve CRF in students. Runningbased protocols with high or maximum intensities similar to recent studies $(16,20,48,50,51)$ seem more appropriate exercise than 6 min Functional ${ }_{\text {HIIT }}$ to improve CRF in students. Most studies showing improved CRF in young individuals incorporate running (21-23) or cycling $(22,24,25)$ based HIIT protocols. It seems these protocols achieve higher stimuli, which trigger cardio-respiratory adaptations more effectively than Functional ${ }_{\mathrm{HIT}}$.

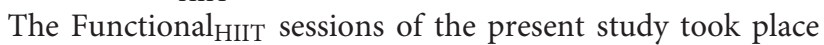
during regular classes and therefore replaced 6 min of sedentary behavior with $6 \mathrm{~min}$ of intensive physical exercise. Consequently,

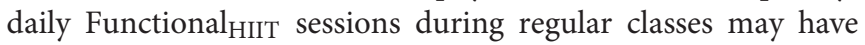
the potential to reduce the amount of sedentary time and may contribute to increase the general level of physical activity in children.

The adherence to the current intervention of Functional was quite high ( $>86 \%$ of all planned sessions), which is not surprising since the exercise program took place during regular classes. Nevertheless, the classroom teacher had no concerns that Functional $_{\text {HIT }}$ session would be disruptive in any form for achieving the curricular aims of the class and therefore approved the intervention beforehand. This high degree of adherence in students and teachers may reflect the fact that the intervention was relatively short and detailed, as well as the fact that the individual sessions did not take much time. In this regards the present study is in line with a very similar study of Ekström et al. (41) who reported that teachers confirmed a good integration of a daily 4 min exercise intervention in the classroom.

Considering the intensity of Functional ${ }_{\mathrm{HIT}}$, students were instructed to perform the Functional ${ }_{\text {HIIT }}$ between 17 and 20 on the Borg scale, whereas 20 represents "all-out" intensity. Since the mean intensity of Functional ${ }_{\text {HIT }}$ sessions was $17 \pm 2$, the RPE scale did reflected the lower range and not the higher range of the intended high-intensity effort.

\section{LIMITATIONS}

A greater number of participants would have given more statistical power to the data interpretation with less risk of calculating type 2 errors. At the same time, the degree to which these results can be generalized is limited due to the small number of participants. The study shows an imbalanced ratio of male and female participants, which represents a limitation since effects of PA interventions may depend on sex in children $(52,53)$. Furthermore, the small sample size does not warrant an analysis for the potential influence of sex or age on the results. However, we included two school classes since (i) the present study represents a first pilot study to test feasibility and adaptions to Functional HIT $_{\text {Th }}$ (ii) a lower sample size allows better controlling of Functional ${ }_{\text {HIT }}$ and increase availability, motivation, and compliance of students during participation.

The inclusion of a physical activity enjoyment scale could have given information on the enjoyment of Functional ${ }_{\text {HIIT }}$ and may had identified if enjoyment of Functional HIT $_{\text {had mediated the }}$ relationship between the exercise program and the outcome.

In order to describe the study participants precisely, some studies applying HIIT with children and/or adolescents (54, 55) incorporate assessment of sexual maturation of participants [e.g., Tanner stages (56)]. Due to personal and organizational restrictions, this was not possible in the present study.

Initially we aimed to record heart rate during the Functional $_{\text {HIT }}$ sessions and we aimed to record Functional ${ }_{\text {HIT }}$ sessions as well as leisure time with accelerometers. However, we refrained of employing this methodology because of the frequent (un)dressing with heart rate straps and the accelerometers.

\section{CONCLUSION}

Four weeks with 3-4 session per week of 6 min Functional $_{\text {HIIT }}$ performed during regular classes do not enhance CRF (assessed by $6 \mathrm{~min}$ test) but improve certain dimensions of functional strength (lateral jumping, sit-ups, 20-m sprint) in 11-year-old students. The Functional ${ }_{\text {HIT }}$ intervention improves 20 -m sprint, lateral jumps and sit-ups to a higher extent compared to a passive in-class control condition. Consequently, the present intervention of Functional ${ }_{\text {HIIT }}$ represents a feasible, sufficient, and effective exercise intervention, which enhances health relevant muscular fitness among young students and could be added as a complement to regular physical education and curricular learning activities during regular classes. As time is limited in the educational system of schools, Functional ${ }_{\text {HIT }}$ during regular school classes may offer a novel and feasible intervention for increasing functional strength in young students. Since Functional ${ }_{\mathrm{HIT}}$ did not improve CRF in children, we recommend performing this type of exercise with greater intensity, longer session duration as well as interventional period.

\section{DATA AVAILABILITY STATEMENT}

The datasets used and/or analyzed during the current study are available from the corresponding author on reasonable request. 


\section{ETHICS STATEMENT}

All procedures were conducted in accordance with the Declaration of Helsinki (30). The experimental protocol was pre-approved by the ethical review board of the Sport Science Institute of the University of Würzburg.

\section{AUTHOR CONTRIBUTIONS}

All authors listed have made a substantial, direct and intellectual contribution to the work, and approved it for publication.

\section{REFERENCES}

1. Van Hecke L, Loyen A, Verloigne M, van der Ploeg HP, Lakerveld J, Brug J, et al. Variation in population levels of physical activity in European children and adolescents according to Cross-European studies: a systematic literature review within DEDIPAC. Int J Behav Nutri Phys Activity. (2016) 13:70. doi: 10.1186/s12966-016-0396-4

2. Hallal PC, Andersen LB, Bull FC, Guthold R, Haskell W, Ekelund U, et al. Global physical activity levels: surveillance progress, pitfalls, and prospects. Lancet. (2012) 380:247-57. doi: 10.1016/S0140-6736(12)60646-1

3. Ortega FB, Ruiz JR, Castillo MJ, Sjöström, M. Physical fitness in childhood and adolescence: a powerful marker of health. Int J Obes. (2008) 32, 1-11. doi: $10.1038 /$ sj.ijo.0803774

4. Blair SN, Kohl HW, Paffenbarger RS, Clark DG, Cooper KH, Gibbons LW. Physical fitness and all-cause mortality. A prospective study of healthy men and women JAMA. (1989) 262:2395-401. doi: 10.1001/jama.1989.03430170057028

5. DeFina LF, William Haskell L, Benjamin Willis L, Carolyn Barlow E, Carrie Finley E, Benjamin Levine D, et al. Physical activity versus cardiorespiratory fitness: two (Partly) distinct components of cardiovascular health? Prog Cardiovasc Dis. (2015) 57:324-29. doi: 10.1016/j.pcad.2014.09.008

6. Suder A, Chrzanowska M. Risk factors for abdominal obesity in children and adolescents from cracow, poland (1983-2000). J Biosoc Sci. (2015) 47:203-19. doi: $10.1017 /$ S0021932013000606

7. WHO. Global Recommendations on Physical Activity for Health. Geneva: World Health Organization (2010).

8. Engel FA, Ackermann A, Chtourou H, Sperlich B. High-intensity interval training performed by young athletes: a systematic review and meta-analysis. Front Physiol. (2018) 9:1012. doi: 10.3389/fphys.2018.01012

9. Costigan SA, Eather N, Plotnikoff RC, Taaffe DR, Lubans DR. Highintensity interval training for improving health-related fitness in adolescents: a systematic review and meta-analysis. Br J Sports Med. (2015) 49:1253-61. doi: 10.1136/bjsports-2014-094490

10. García-Hermoso A, Cerrillo-Urbina A, Herrera-Valenzuela JT, CristiMontero C, Saavedra JM, Martínez-Vizcaíno V. Is high-intensity interval training more effective on improving cardiometabolic risk and aerobic capacity than other forms of exercise in overweight and obese youth? a meta-analysis. Obes Rev. (2016). 17:531-40. doi: 10.1111/obr.12395

11. Braaksma P, Stuive I, Garst RME, Wesselink CF, van der Sluis CK, Dekker $\mathrm{R}$, et al. Characteristics of physical activity interventions and effects on cardiorespiratory fitness in children aged 6-12 years-A systematic review. J Sci Med Sport. (2018) 21:296-306. doi: 10.1016/j.jsams.2017.07.015

12. Eddolls WTB, McNarry MA, Stratton G, Winn CON, Mackintosh KA. High-intensity interval training interventions in children and adolescents: a systematic review. Sports Med. (2017) 47:2363-74. doi: 10.1007/s40279-017-0753-8

13. Thivel D, Masurier J, Baquet G, Timmons BW, Pereira B, Berthoin S, et al. High-intensity interval training in overweight and obese children and adolescents: systematic review and meta-analysis. J Sports Med Phys Fitness. (2018) 59:310-24. doi: 10.23736/S0022-4707.18.08075-1

\section{FUNDING}

We acknowledge financial support by German Research Foundation (DFG) within the funding programme Open Access Publishing, by the Baden-Württemberg Ministry of Science, Research and the Arts and by Ruprecht-KarlsUniversität Heidelberg.

\section{ACKNOWLEDGMENTS}

The authors thank the students for their enthusiastic participation as well as the teachers and the undergraduate students for their assistance and support.

14. Fox KR, Cooper A, McKenna J. The school and promotion of children's health-enhancing physical activity: perspectives from the United Kingdom. J Teach Phys Educ. (2004) 23:338-58. doi: 10.1123/jtpe.23.4.338

15. Morris A, Cramb R, Dodd-Reynolds CJ. Food intake and appetite following school-based high-intensity interval training in 9-11-year-old children. $J$ Sports Sci. (2018) 36:286-92. doi: 10.1080/02640414.2017.1302599

16. Delgado-Floody P, Espinoza-Silva M, García-Pinillos F, Latorre-Román P. Effects of 28 weeks of high-intensity interval training during physical education classes on cardiometabolic risk factors in chilean schoolchildren: a pilot trial. Eur J Pediatr. (2018) 177:1019-27. doi: 10.1007/s00431-0183149-3

17. Weston KL, Azevedo LB, Bock S, Weston M, George KP, Batterham AM. Effect of novel, school-based high-intensity interval training (HIT) on cardiometabolic health in adolescents: project FFAB (fun fast activity blasts) - an exploratory controlled before-and-after trial. PLoS ONE. (2016) 11:e0159116. doi: 10.1371/journal.pone.0159116

18. Leahy AA, Eather N, Smith JJ, Hillman CH, Morgan PJ, Plotnikoff RC, et al. Feasibility and preliminary efficacy of a teacher-facilitated high-intensity interval training intervention for older adolescents. Pediatr Exercise Sci. (2018) 31:107-17. doi: 10.1123/pes.2018-0039

19. Buchan DS, Young JD, Simpson AD, Thomas NE, Cooper SM, Baker JS. The effects of a novel high intensity exercise intervention on established markers of cardiovascular disease and health in scottish adolescent youth. J Public Health Res. (2012) 1:155-57. doi: 10.4081/jphr.2012.e24

20. Baquet G, Berthoin S, Gerbeaux M, Van Praagh E. High-intensity aerobic training during a 10 week one-hour physical education cycle: effects on physical fitness of adolescents aged 11 to 16. Int J Sports Med. (2001) 22:295300. doi: $10.1055 / \mathrm{s}-2001-14343$

21. McManus AM, Cheng $\mathrm{CH}$, Leung MP, Yung TC, Macfarlane DJ. Improving aerobic power in primary school boys: a comparison of continuous and interval training. Int J Sports Med. (2005) 26:781-86. doi: $10.1055 / \mathrm{s}-2005-837438$

22. Gutin B, Barbeau P, Owens S, Lemmon CR, Bauman M, Allison J, et al. Effects of exercise intensity on cardiovascular fitness, total body composition, and visceral adiposity of obese adolescents. Am J Clin Nutri. (2002) 75:818-26. doi: 10.1093/ajcn/75.5.818

23. Buchan DS1, Ollis S, Young JD, Cooper SM, Shield JP, Baker JS. High intensity interval running enhances measures of physical fitness but not metabolic measures of cardiovascular disease risk in healthy adolescents. BMC Public Health. (2013) 13:498. doi: 10.1186/1471-2458-13-498

24. Breil FA, Weber SN, Koller S, Hoppeler H, Vogt M. Block training periodization in alpine skiing: effects of 11-day HIT on VO2max and performance. Eur J Appl Physiol. (2010) 109:1077-86. doi: 10.1007/s00421-010-1455-1

25. Boer PH1, Meeus M, Terblanche E, Rombaut L, Wandele ID, Hermans L, et al. The influence of sprint interval training on body composition, physical and metabolic fitness in adolescents and young adults with intellectual disability: a randomized controlled trial. Clin Rehabilitation. (2014) 28, $221-$ 31. doi: $10.1177 / 0269215513498609$ 
26. Sperlich B, Hahn LS, Edel A, Behr T, Helmprobst J, Leppich R, et al. A 4Week Intervention Involving Mobile-Based Daily 6-Minute Micro-Sessions of Functional High-Intensity Circuit Training Improves Strength and Quality of Life, but Not Cardio-Respiratory Fitness of Young Untrained Adults. Front Physiol. (2018) 9:92. doi: 10.3389/fphys.2018.00423

27. Sperlich B, Wallmann-Sperlich B, Zinner C, Von Stauffenberg V, Losert H, Holmberg HC. Functional high-intensity circuit training improves body composition, peak oxygen uptake, strength, and alters certain dimensions of quality of life in overweight women. Front Physiol. (2017) 8:172. doi: 10.3389/fphys.2017.00172

28. Júdice PB, Silva AM, Berria J, Petroski EL, Ekelund U, Sardinha LB. Sedentary patterns, physical activity and health-related physical fitness in youth: a cross-sectional study. Int J Behav Nutri Phys Activity. (2017) 14:25. doi: 10.1186/s12966-017-0481-3

29. Godin G, Desharnais R, Valois P, Lepage L, Jobin J, Bradet R. Differences in perceived barriers to exercise between high and low intenders: observations among different populations. Am J Health Promot. (1994) 8:279-85. doi: $10.4278 / 0890-1171-8.4 .279$

30. World Medical Association. Ethical principles for medical research involving human subjects. JAMA. (2013) 310:2191-4. doi: 10.1001/jama.2013.281053

31. Bös K, Tittlbach S. Deutscher Motorik-Test 6-18 (DMT 6-18). Hamburg: Feldhaus (2016).

32. Borg G. Perceived Exertion as an Indicator of Somatic Stress. Scand J Rehabilitation Med. (1970) 2:92-8. doi: 10.1037/t58166-000

33. Williams JG, Eston RG, Stretch C. Use of the rating of perceived exertion to control exercise intensity in children. Pediatr Exercise Sci. (1991) 3:21-7. doi: $10.1123 /$ pes.3.1.21

34. Spengler S, Rabel M, Kuritz AM, Mess F. Trends in motor performance of first graders: a comparison of cohorts from 2006 to 2015. Front. Pediatr. (2017) 5:206. doi: 10.3389/fped.2017.00206

35. Santner A, Kopp M, Federolf P. Partly randomised, controlled study in children aged 6-10 years to investigate motor and cognitive effects of a 9-week coordination training intervention with concurrent mental tasks. BMJ Open. (2018) 8:e021026. doi: 10.1136/bmjopen-2017-021026

36. König TT, Muensterer OJ. Physical fitness and locomotor skills in children with esophageal atresia-a case control pilot study. Front Pediatr. (2018) 6:337. doi: 10.3389/fped.2018.00337

37. Gillach MC, Sallis JF, Buono MJ, Patterson P, Nader PR. The relationship between perceived exertion and heart rate in children and adults. Ped Exer Sci. (1989) 1:360-8. doi: 10.1123/pes.1.4.360

38. Richardson JTE. Eta squared and partial eta squared as measures of effect size in educational research. Educ Res Rev. (2011) 6:135-47. doi: 10.1016/j.edurev.2010.12.001

39. Clark-Carter D. Doing Quantitative Psychological Research: From Design to Report. Erlbaum: Psychology Press Taylor \& Francis (1997).

40. Faul F, Erdfelder E, Lang AG, Buchner AG. G*Power 3: a flexible statistical power analysis program for the social, behavioral, and biomedical sciences. Behav Res Methods. (2007) 2007:175-91. doi: 10.3758/BF 03193146

41. Ekström A, Östenberg AH, Björklund G, Alricsson M. The effects of introducing tabata interval training and stability exercises to school children as a school-based intervention program. Int J Adolesc Med Health. (2017) 31. doi: 10.1515/ijamh-2017-0043

42. Costigan SA, Eather N, Plotnikoff RC, Taaffe DR, Pollock E, Kennedy SG, et al. Preliminary efficacy and feasibility of embedding high intensity interval training into the school day: a pilot randomized controlled trial. Prev Med Rep. (2015) 2:973-9. doi: 10.1016/j.pmedr.2015.11.001

43. Ozmun JC, Mikesky AE, Surburg PR. Neuromuscular adaptations following prepubescent strength training. Med Sci Sports Exercise. (1994) 26:510-14. doi: 10.1249/00005768-199404000-00017
44. Granacher U, Goesele A, Roggo K, Wischer T, Fischer S, Zuerny C, et al. Effects and mechanisms of strength training in children. Int J Sports Med. (2011) 32:357-64. doi: 10.1055/s-0031-1271677

45. Peitz M, Behringer M, Granacher U. A systematic review on the effects of resistance and plyometric training on physical fitness in youthWhat do comparative studies tell us? PLoS ONE. (2018) 13:e0205525. doi: 10.1371/journal.pone.0205525

46. Behm DG, Young JD, Whitten JHD, Reid JC, Quigley PJ, Low J, et al. Effectiveness of traditional strength vs. power training on muscle strength, power and speed with youth: a systematic review and meta-analysis. Front Physiol. (2017) 8:423. doi: 10.3389/fphys.2017.00423

47. Granacher U, Muehlbauer T, Doerflinger B, Strohmeier R, Gollhofer A. promoting strength and balance in adolescents during physical education: effects of a short-term resistance training. J Strength Condition Res. (2011) 25:940-49. doi: 10.1519/JSC.0b013e3181c7bb1e

48. van Biljon A, McKune AJ, DuBose KD, Kolanisi U, Semple SJ. Do shortterm exercise interventions improve cardiometabolic risk factors in children? J Pediatri. (2018) 203:325-9. doi: 10.1016/j.jpeds.2018.07.067

49. Buchheit M, Laursen PB. High-intensity interval training, solutions to the programming puzzle: part i: cardiopulmonary emphasis. Sports Med. (2013) 43:313-38. doi: 10.1007/s40279-013-0029-x

50. Engel F, Wagner M, Roth A, Scharenberg S, Bossmann T, Woll A, et al. Hochintensives Intervalltraining im Sportunterricht. Ger J Exerc Sport Res. (2018) 48:120-28. doi: 10.1007/s12662-018-0492-5

51. Weiss MR, Phillips AC, Kipp LE. Effectiveness of a school-based fitness program on youths' physical and psychosocial health outcomes. Pediatr Exercise Sci. (2015) 27:546-57. doi: 10.1123/pes.2015-0011

52. Lazaar N, Aucouturier J, Ratel S, Rance M, Meyer M, Duché P. Effect of physical activity intervention on body composition in young children:influence of body mass index status and gender. Acta Paediatr. (2007) 96:1315-20. doi: 10.1111/j.1651-2227.2007.00426.x

53. Martínez-Vizcaíno V, Sánchez-López M, Notario-Pacheco B, Salcedo-Aguilar F, Solera-Martínez M, Franquelo-Morales P, et al. Gender differences on effectiveness of a school-based physical activity intervention for reducing cardiometabolic risk: a cluster randomized trial. Int J Behav Nutr Phys Acta. (2014) 11:154. doi: 10.1186/s12966-014-0154-4

54. Corte de Araujo, A. C., Roschel, H., Picanço, A. R., do Prado, D. M., Villares, S. M., de Sá Pinto, A. L., et al. Similar health benefits of endurance and high-intensity interval training in obese children. PLoS ONE. (2012) 7:e42747. doi: 10.1371/journal.pone.0042747

55. Sperlich B, Zinner C, Heilemann I, Kjendlie P-L, Holmberg H-C, Mester J. High-intensity interval training improves VO2peak, maximal lactate accumulation, time trial and competition performance in 9-11-year-old swimmers. Eur J Appl Physiol. (2010) 110:1029-36. doi: 10.1007/s00421-010-1586-4

56. Tanner JM, Whitehouse RH. Clinical longitudinal standards for height, weight, height velocity, weight velocity, and stages of puberty. Arch Dis Child. (1976) 51:170-9. doi: 10.1136/adc.51.3.170

Conflict of Interest: The authors declare that the research was conducted in the absence of any commercial or financial relationships that could be construed as a potential conflict of interest.

Copyright (C) 2019 Engel, Wagner, Schelhorn, Deubert, Leutzsch, Stolz and Sperlich. This is an open-access article distributed under the terms of the Creative Commons Attribution License (CC BY). The use, distribution or reproduction in other forums is permitted, provided the original author(s) and the copyright owner(s) are credited and that the original publication in this journal is cited, in accordance with accepted academic practice. No use, distribution or reproduction is permitted which does not comply with these terms. 